\title{
Alkali-Cation Affinities of Polyoxyethylene Dodecylethers and Helical Conformations of Their Cationized Molecules Studied by Electrospray Mass Spectrometry
}

\author{
Yukio Yokoyama, ${ }^{a}$ Rui Hirajima, ${ }^{\text {a }}$ Ken Morigaki, ${ }^{a}$ \\ Yoshitaka Yamaguchi, ${ }^{b}$ and Kazuyoshi Ueda ${ }^{b}$ \\ ${ }^{a}$ Department of Analytical Chemistry, Faculty of Engineering, Yokohama National University, Yokohama, \\ Japan \\ b Department of Material Science, Faculty of Engineering, Yokohama National University, Yokohama, Japan
}

\begin{abstract}
Relative alkali-cation affinity of polyoxyethylene (POE) dodecylethers in gas phase was studied by electrospray ionization (ESI) mass spectrometry using dodecylether-poly-ethoxylate $\left(\mathrm{C}_{12} \mathrm{EO}: n\right.$, " $n$ " denotes ethyleneoxide unit number) nonionic surfactants, and possible helical conformations of the cationized molecules were demonstrated. The alkali-cation affinity highly depended on the cation diameters. The mass spectra of $\mathrm{C}_{12} \mathrm{EO}: 8$ cationized by alkali-metal ions were dominated by potassiated molecules. The results indicated that the POE moiety could have specific affinity to $\mathrm{K}^{+}$ions based on a host-guest interaction between POE helix and potassium ions. This is very similar to the relationships between 18 -crown- 6 and $\mathrm{K}^{+}$. The ESI mass spectra exhibited the multiply cationized $\mathrm{C}_{12} \mathrm{EO}: n$ in addition to the singly cationized molecules. The critical EO unit numbers necessary for producing the multiply-charged cationized molecules also depended on the cation diameters. In addition, the POE surfactants highly preferred alkali cations to proton. The results were strongly supported by molecular mechanics/dynamics calculations. A helical conformation of the POE moiety of $\mathrm{C}_{12} \mathrm{EO}: 15$ including two $\mathrm{K}^{+}$ions gave a potential minimum, while a lowest energy structure of the protonated molecule took irregular conformations due to the formation of local hydrogen bonds. (J Am Soc Mass Spectrom 2007, 18, 1914-1920) (c 2007 American Society for Mass Spectrometry
\end{abstract}

$\mathrm{E}$ lectrospray ionization (ESI) [1] mass spectrometry can often provide alkali-cation adducts for organic compounds in addition to producing their protonated molecules. In our previous work analyzing unknown polyoxyethylene nonionic surfactants, the ESI mass spectra have exhibited their intense $\mathrm{Na}^{+}$or $\mathrm{K}^{+}$adducts rather than their protonated molecules [2]. Our primary interest arose from the ESI mass spectral results, in which the peak distribution of the surfactants observed as $\mathrm{K}^{+}$adducts, due to the polyoxyethylene (POE) chain-length distribution, was very different from those observed as $\mathrm{Na}^{+}$adducts. Despite the same surfactant, in other words, the chain length of the POE homologues giving peak maxima as $\mathrm{K}^{+}$adducts, were significantly different form those giving as $\mathrm{Na}^{+}$adducts in the ESI mass spectra. It is thus estimated that the longer POE chains may have specific and selective affinity to alkali cations. On the other hand, a molecular modeling simulation study has proposed an interesting result that poly(ethylene glycol)s (PEGs) can give helical conformations in the aqueous phase [3]. According

Address reprint requests to Dr. Y. Yokoyama, Department of Analytical Chemistry, Yokohama National University, 79-5 Tokiwadai, Hodogaya, Yokohama 240-8501, Japan. E-mail: yokyuk@ynu.ac.jp to the paper, the calculation has suggested that one helical turn is comprised of four consecutive oxyethylene units. From this, we thus consider that the helical conformation, allowing oxygen atoms toward inside, can include alkali-metal cations inside the helix as well as the fact that the cyclic polyethers (crown ethers) can give specific interaction to alkali-metal cations [4-12].

Copolymers of ethylene oxide (EO) and their related compounds are important materials in modern industrial surfactant manufacturing. In addition to this, PEGs are often used as the $\mathrm{m} / \mathrm{z}$ calibration materials in the atmospheric pressure ionization mass spectrometers such as ESI-MS. Therefore, the mass spectral characteristics of their alkali-cation adducts have been well studied by many workers [13-18]. The formation of multiply charged molecules cationized by alkali-metal ions is widely recognized but the differences in alkalication affinity have been still unclear. In addition to this, gas-phase conformations of PEGs and/or related copolymers associated with alkali cations have also been presented [19-22]. Although the molecular structures of cationized EO oligomers have been characterized by experimental and/or theoretical methods, we can find few data showing helical conformation of PEGs. 
This paper describes relative alkali-metal cation affinity of dodecylether-[n-mole]-ethoxylates, referred to as $\mathrm{C}_{12} \mathrm{EO}: n$, which are directly compared with those of cyclic polyethers (i.e., crown ethers), by means of ESI mass spectrometry. Stable helical conformations of cationized $\mathrm{C}_{12} \mathrm{EO}: n$ in the gas phase are proposed from the ESI mass spectral results, and the estimated conformations of the cationized molecules are well demonstrated by molecular mechanics/dynamics calculations using several model $\mathrm{C}_{12} \mathrm{EO}: n$ compounds.

\section{Experimental}

\section{Reagents}

All alkali metal iodides (LiI, NaI, KI, RbI, and CsI), and trifluoroacetic acid (TFA) were purchased from Wako (Osaka, Japan). Pentaoxyethylenedodecylether (dodecylether-5 mol-ethoxylate, referred to as $\mathrm{C}_{12} \mathrm{EO}: 5, \mathrm{EO}$ denotes ethylene oxide unit, MW: 406), hexaoxyethylenedodecylether (dodecylether-6 mol-ethoxylate, $\mathrm{C}_{12} \mathrm{EO}: 6, \mathrm{MW}$ : 450), heptaoxyethylenedodecylether (dodecylether-7 molethoxylate, $\mathrm{C}_{12} \mathrm{EO}: 7, \mathrm{MW}: 494$ ), and octaoxyethylenedodecylether (dodecylether-8 mol-ethoxylate, $\mathrm{C}_{12} \mathrm{EO}: 8, \mathrm{MW}$ : 538, (Scheme 1) were purchased from Sigma (St. Louis, MO) or Wako. These surfactants have no distribution in the degree of oxyethylene polymerization. Other synthetic dodecylether- $n$ mol-ethoxylate, referred to as $\mathrm{C}_{12} \mathrm{EO}$ : $n$ (average) of averaged EO polymerization number $=7$, $13,16,20$, and 40, were furnished by ADEKA (Tokyo, Japan). 12-Crown-4 (MW: 176), 15-crown-5 (MW: 220), and 18-crown-6 (MW: 264) were purchased from Wako. Methanol of HPLC grade was purchased from Kanto Kagaku (Tokyo, Japan). All reagents were used without further purification. Water was purified by passing through a Nihon Millipore (Tokyo, Japan) Direct-Q water purification system just before use.

\section{Instrumentation}

A Shimadzu (Kyoto, Japan) LC-MS QP8000 liquid chromatograph/quadrupole mass spectrometer equipped with an electrospray ionization source was used for the measurements, which was operated like a flowinjection system without any separation columns. The mobile phase of $50 \%$ (vol/vol) methanol/water mixture was delivered to the ion source at a flow rate of 0.1 $\mathrm{mL} / \mathrm{min}$. The instrumental conditions were automatically tuned with a polyethyleneglycol 600 solution as a tuning standard, typically as follows: probe voltage, $+4.5 \mathrm{kV}$; skimmer cone voltage, $+45 \mathrm{~V}$; nebulizing gas $\left(\mathrm{N}_{2}\right), 4.5 \mathrm{~L} / \mathrm{min}$; CDL capillary (located between ion source and skimmer cone) temperature, $230{ }^{\circ} \mathrm{C}$; CDL voltage, $-31 \mathrm{kV}$; and detector gain, $-2 \mathrm{kV}$. The mass

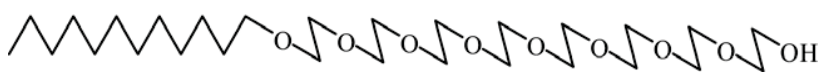

Scheme 1. A schematic structure of dodecylether-8 mol-ethoxylate $\left(\mathrm{C}_{12} \mathrm{EO}: 8\right)$. analyzer was full-scanned at $4000 \mathrm{u} / \mathrm{s}$ in positive mode, scanning over the range between $\mathrm{m} / \mathrm{z} 50$ and 1000. The sample size was $5 \mu \mathrm{L}$ in all injections.

\section{Sample Preparation}

Stock solutions of $20 \mathrm{mM} \mathrm{C}_{12} \mathrm{EO}: 5,6,7$, and 8 were prepared by dissolving a $1 \mathrm{mmol}$ of each authentic reagent into $50 \mathrm{~mL}$ of $50 \%$ (vol/vol) methanol-water mixture. A stock solution of $20-\mathrm{mM}$ crown ether was also prepared in each by the same way. Similarly, a stock solution of $1 \%$ (wt/vol) highly ethoxylated $\mathrm{C}_{12} \mathrm{EO}: n$ (average) was prepared by dissolving a $50 \mathrm{mg}$ of each synthetic surfactant into $5 \mathrm{~mL}$ of $50 \%$ (vol/vol) methanol-water mixture. A $10-\mathrm{mM}$ alkali metal iodide in $50 \%$ (vol/vol) methanol-water was prepared in each.

(1) $\mathrm{C}_{12} \mathrm{EO}: n$-alkali-metal ion mixtures: A $10-\mu \mathrm{L}$ aliquot of the 20-mM C ${ }_{12}$ EO: $n$ was diluted individually 100 times by adding a $990-\mu \mathrm{L}$ aliquot of each $10-\mathrm{mM}$ cation solution, which led to $\sim 50$ times molar excess of alkali-metal ion to the individual $\mathrm{C}_{12} \mathrm{EO}: n$ (5-8). The final solution ready for injection was $\sim 0.2 \mathrm{mM} \mathrm{C}_{12} \mathrm{EO}: n$ in $10 \mathrm{mM}$ cation(s). In the same way, a 100- $\mu \mathrm{L}$ aliquot of the highly ethoxylated $\mathrm{C}_{12} \mathrm{EO}: n$ (average) was mixed with a $900-\mu \mathrm{L}$ aliquot of $10-\mathrm{mM}$ cation solution, which led to the 10 times dilution and the 5 times molar excess of cation.

(2) $\mathrm{C}_{12} \mathrm{EO}: n$ (5-8)-crown-ether-alkali-metal ion mixtures: A $100-\mu \mathrm{L}$ aliquot of one of the pure $\mathrm{C}_{12} \mathrm{EO}: n$ and $\mathrm{a}$ $100-\mu \mathrm{L}$ aliquot of one of the crown ethers $(12,15$, and 18 crowns) were dissolved in a $800-\mu \mathrm{L}$ aliquot of one of the alkali-metal ion solutions, which led to 10 times dilution for individual polyether compounds and 5 times molar excess of one of alkali cations $\left(\mathrm{Li}^{+}, \mathrm{Na}^{+}\right.$, and $\mathrm{K}^{+}$). In the same way, the samples ready for injection in several different combinations were prepared.

\section{Molecular Modeling Simulation}

Molecular mechanics/dynamics calculations were performed using CHARMM29 (Chemistry at Harvard Macromolecular Mechanics) program [23]. The force field for polyoxyethylene developed by Tasaki [3], having developed for optimizing helical conformations of POE in aqueous phase in consideration of water molecules, was applied to the present gas-phase calculations. Since the parameters for the hydroxyl endresidue have not been included in Tasaki's set, those parameters were taken from the CHARMM29 parameter set. The conformational optimization was performed on the model POE surfactants of $\mathrm{C}_{12} \mathrm{EO}: 7,12$, and 15 with and without including $\mathrm{Na}^{+}, \mathrm{K}^{+}$, or $\mathrm{H}^{+}$. Several initial structures of the polyoxyethylenedodecylether were constructed by changing the dihedral angles of $\mathrm{OC}-\mathrm{CO}$ and $\mathrm{CO}-\mathrm{CC}$, and then they were minimized to investigate the lowest energy conformation. The basic initial structure was taken from a helical structure, which has $\mathrm{OC}-\mathrm{CO}$ and $\mathrm{CO}-\mathrm{CC}$ of gauche and trans, 

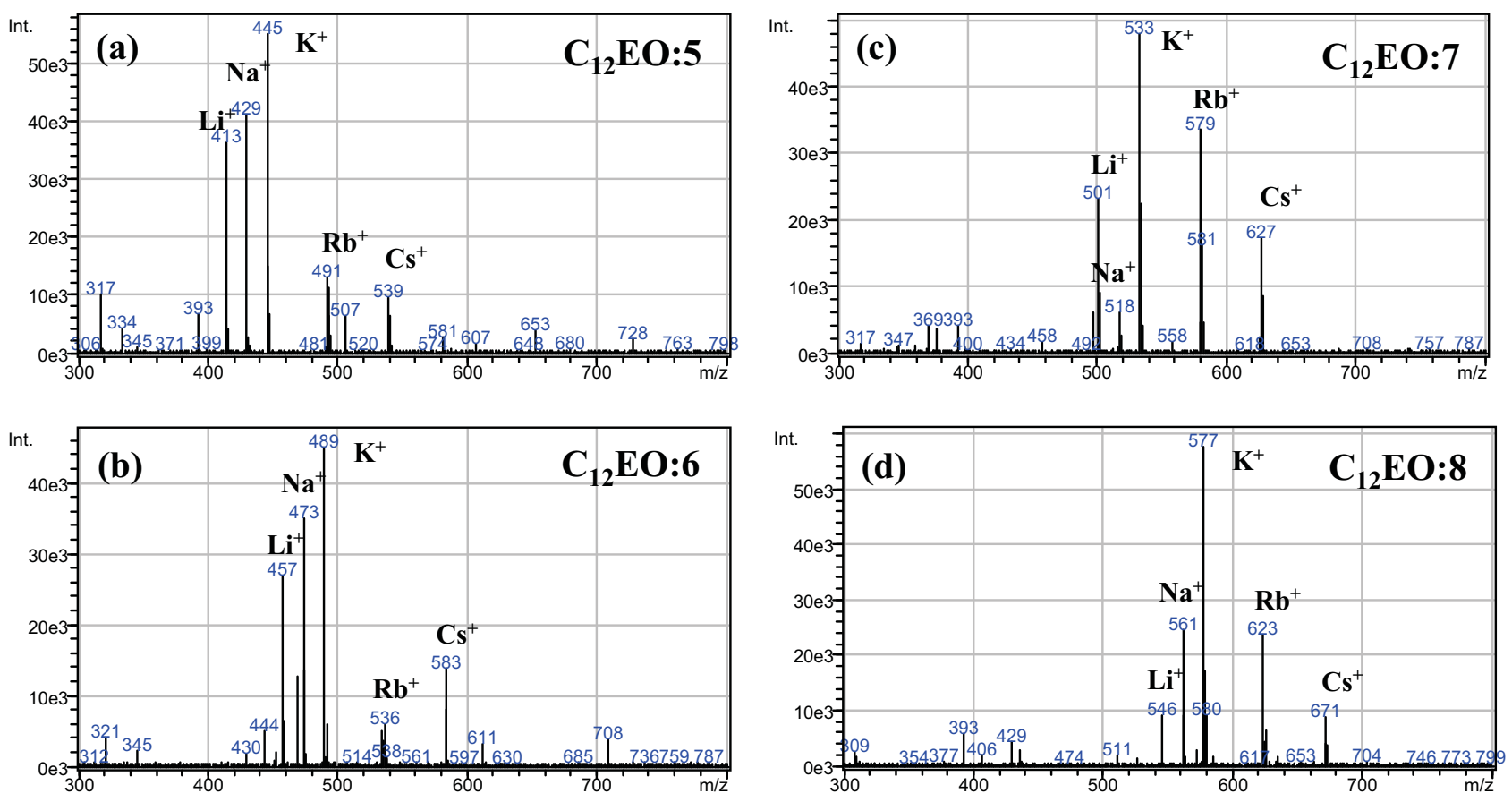

Figure 1. ESI mass spectra of $\mathrm{C}_{12} \mathrm{EO}: n$ cationized by alkali cations. (a) $\mathrm{C}_{12} \mathrm{EO}: 5\left(\mathrm{~m} / z 413[\mathrm{M}+\mathrm{Li}]^{+}\right.$; $\left.m / z 429[\mathrm{M}+\mathrm{Na}]^{+} ; m / z 445[\mathrm{M}+\mathrm{K}]^{+} ; m / z 491[\mathrm{M}+\mathrm{Rb}]^{+} ; m / z 539[\mathrm{M}+\mathrm{Cs}]^{+}\right),(\mathbf{b}) \mathrm{C}_{12} \mathrm{EO}: 6(\mathrm{~m} / z 457$ $\left.[\mathrm{M}+\mathrm{Li}]^{+} ; m / z 473[\mathrm{M}+\mathrm{Na}]^{+} ; m / z 489[\mathrm{M}+\mathrm{K}]^{+} ; m / z 535[\mathrm{M}+\mathrm{Rb}]^{+} ; m / z 583[\mathrm{M}+\mathrm{Cs}]^{+}\right),(\mathrm{c}) \mathrm{C}_{12} \mathrm{EO}: 7$ $\left(m / z 501[\mathrm{M}+\mathrm{Li}]^{+} ; m / z 517[\mathrm{M}+\mathrm{Na}]^{+} ; m / z 533[\mathrm{M}+\mathrm{K}]^{+} ; m / z 579[\mathrm{M}+\mathrm{Rb}]^{+} ; m / z 627[\mathrm{M}+\mathrm{Cs}]^{+}\right)$, (d) $\mathrm{C}_{12} \mathrm{EO}: 8\left(\mathrm{~m} / z 545[\mathrm{M}+\mathrm{Li}]^{+} ; \mathrm{m} / z 561[\mathrm{M}+\mathrm{Na}]^{+} ; m / z 577[\mathrm{M}+\mathrm{K}]^{+} ; m / z 623[\mathrm{M}+\mathrm{Rb}]^{+} ; m / z 671\right.$ $\left.[\mathrm{M}+\mathrm{Cs}]^{+}\right)$.

respectively, [3]. Then, several other initial structures were constructed by changing the dihedral angles in the range of $\pm 30^{\circ}$ from the basic helical structure.

\section{Results and Discussion}

\section{Relative Cation Affinity of POE Oligomers}

Figure 1 shows typical ESI mass spectra of (a) $\mathrm{C}_{12} \mathrm{EO}: 5$, (b) $\mathrm{C}_{12} \mathrm{EO}: 6$, (c) $\mathrm{C}_{12} \mathrm{EO}: 7$, and (d) $\mathrm{C}_{12} \mathrm{EO}: 8$ measured with addition of five kinds of alkali-metal cations, which look well indicating the difference in cation affinity of the POE surfactants. Since the mole of each cation added to the sample was equally excessive to the individual surfactants, the relative peak intensity of the cationized molecules observed in the mass spectra could show the relative cation affinities. This resemblance of the mass spectra between $\mathrm{C}_{12} \mathrm{EO}: 5$ (Figure 1a) and $\mathrm{C}_{12} \mathrm{EO}: 6$ (Figure $1 \mathrm{~b}$ ) may indicate that the $\mathrm{C}_{12} \mathrm{EO}: n$ surfactants having smaller EO unit number less than $n=6$ can have similar affinity to the alkali cations. The potassiated molecules $\left([\mathrm{M}+\mathrm{K}]^{+}\right)$dominated in all mass spectra. This can suggest that the potassium-ion affinity of the POE oligomers is significantly high and probably independent of the chain length, which can lead to the estimation that the potassium ions can fit the POE helical cavity by size matching. This is very similar to the well known fact that potassium ion can be included selectively in the electron-rich hole of 18crown- 6 by size fitting [26]. In contrast, the cesiated
Table 1. Ionic diameters of alkali cations [24] and hole diameters of crown ethers [25]

\begin{tabular}{cccc}
\hline Alkali cation & $\begin{array}{c}\text { lonic diameter } \\
(\mathrm{pm})\end{array}$ & Crown ether & $\begin{array}{c}\text { Hole diameter } \\
(\mathrm{pm})\end{array}$ \\
\hline \hline $\mathrm{Li}^{+}$ & 120 & $12-$ crown-4 & $120-150$ \\
$\mathrm{Na}^{+}$ & 190 & $15-$ crown-5 & $170-220$ \\
$\mathrm{~K}^{+}$ & 266 & $18-$ crown-6 & $260-320$ \\
$\mathrm{Rb}^{+}$ & 296 & & \\
$\mathrm{Cs}^{+}$ & 338 & & \\
\hline
\end{tabular}

molecules $\left([\mathrm{M}+\mathrm{Cs}]^{+}\right)$were relatively less abundant than other cationized molecules, probably because of too large ionic diameter.

Figure 2a shows the relative abundances of cationized molecules of $\mathrm{C}_{12} \mathrm{EO}: n$ versus cation diameters listed in Table 1, summarized by adding up the peak intensity data from the individual mass spectra such as shown in Figure 1. Although there was some difference in the ion abundance, each alkali-metal cation could produce the cationized $\mathrm{C}_{12} \mathrm{EO}: n$ (5-8), probably by forming a stable helical conformation including cation. As can be seen in Figure 2a, the longer POE chain can include the larger cations.

On the other hand, the ESI mass spectra of crown ethers exhibited the individual alkali-cationized molecules, which were very specific compared with those of cationized $\mathrm{C}_{12} \mathrm{EO}: n$. Figure $2 \mathrm{~b}$ shows the relative ionic abundances of the cationized crown ethers versus the 

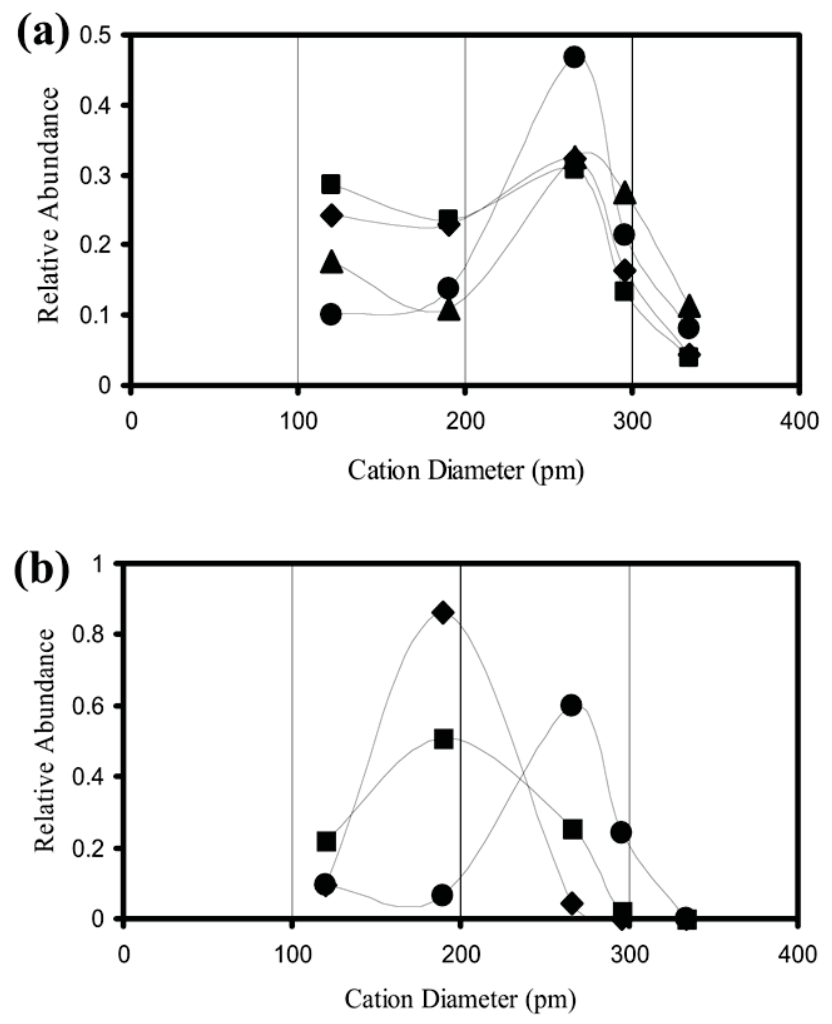

Figure 2. (a) Relative ionic abundances of $\mathrm{C}_{12} \mathrm{EO}: 5(\bullet), 6(\mathbf{\square}), 7$ $(\boldsymbol{\Delta})$, and $8(\mathbf{O})$ cationized by alkali-metal ions. (b) Relative ionic abundances of 12-Crown-4 $(\boldsymbol{\nabla}), 15-C r o w n-5(\boldsymbol{\square})$, and 18-Crown-6 (๑) cationized by alkali-metal ions. The cation diameters (horizontal axis) are referable to Table 1.

cation diameters. Although $\mathrm{C}_{12} \mathrm{EO}: n$ (5-8) could give significantly abundant their cesiated molecules ([M + $\mathrm{Cs}]^{+}$), as seen in Figure 2a, 18-crown-6 produced less abundant cesiated molecules. Similarly, 15-crown-5 and 12-crown-4 both exhibited less intense peaks of their $[\mathrm{M}+\mathrm{Rb}]^{+}$and $[\mathrm{M}+\mathrm{Cs}]^{+}$. 18-Crown-6 showed the specific selectivity to potassium ions, and similarly both 15-crown-5 and 12-crown-4 showed the specificity to sodium ions. The results can suggest that the POE chains are not so selective to alkali cations, probably because of forming flexible helical structures, while the cyclic POEs are very selective because of their rigid ring structures. The hole diameters of the crown ethers examined in this work are too small to hold larger cations such as $\mathrm{Rb}^{+}$and $\mathrm{Cs}^{+}$(see Table 1).

Clearly the cation affinity of the POE oligomers is very similar to that of 18 -crown-6. In other words, the sectional diameter toward the axis of the helical POE chain is very close to the hole diameter of 18-crown-6. The big difference between them is that the crown ether can include the best-fitting cation into the hole but POE chain can change the helical conformation according to the size of the guest cation. Since the pitch distance of the helical turn seems to be variable or flexible in accordance with the diameter of the cation, the $\mathrm{C}_{12} \mathrm{EO}: n$ (5-8) can include larger $\mathrm{Rb}^{+}$and $\mathrm{Cs}^{+}$ions (Figure 2a) but 18-crown-6 can not include such too large cations (Figure 2b).

\section{Comparison of Cation Affinities Between Helical and Cyclic Polyethers}

In the cases coexisting $\mathrm{C}_{12} \mathrm{EO}: n$ and crown ether in equal molar concentration, each mass spectrum was dominated by the cationized $\mathrm{C}_{12} \mathrm{EO}: n$ rather than cationized crown ether. This can indicate that the cation affinity of helical POE is significantly higher than that of cyclic POE, although the cation selectivity of crown ether was significantly higher than that of POE surfactants as described above (Figure 2). The $\mathrm{K}^{+}$ affinity of $\mathrm{C}_{12} \mathrm{EO}: 5$ was 2.5 times higher than that of 18-crown-6, both having six oxygen atoms. The potassiated $\mathrm{C}_{12}$ EO:8 was about five times more stable than the potassiated 18-crown-6. In addition, significant peaks of potassiated 12-crown-4 were not observed in every mass spectrum, probably because the $\mathrm{K}^{+}$affinity of 12-crown- 4 was considerably less than that of POE. Similarly, the $\mathrm{Na}^{+}$and $\mathrm{Li}^{+}$affinities of $\mathrm{C}_{12}$ EO:n (5-8) were $1.7 \sim 10$ times or more higher than those of crown ethers.

The clear and significant difference in cation affinities between $\mathrm{C}_{12}$ EO:n (5-8) and crown ether is probably owing to the difference in flexibility between their stereo structures. The POE chains can change their helical pitches flexibly according to the cation size, leading to a stable conformation. In contrast to this, cyclic polyethers having less flexible ring structure can provide high specificity to alkali cations due to their particular size selectivity.

\section{Critical EO Unit Number Necessary for Forming Multiply Charged Cationized Molecules}

As the EO unit number increased, the ESI mass spectra could exhibit the multiply charged $\mathrm{C}_{12} \mathrm{EO}: n$ (average) cationized by alkali metal ions in addition to their singly cationized molecules. Figure 3 shows the typical mass spectra of (a) $\mathrm{C}_{12} \mathrm{EO}: 13$ (average) and (b) $\mathrm{C}_{12} \mathrm{EO}$ : 40 (average) both cationized by $\mathrm{K}^{+}$, in which two series of peaks can be recognized clearly between $\mathrm{m} / \mathrm{z} 300$ and 1000. Figure $3 a$ exhibited the serial peaks of singly potassiated $\mathrm{C}_{12} \mathrm{EO}: n(2-17)$ with $44 \mathrm{u}$ intervals and doubly potassiated $\mathrm{C}_{12} \mathrm{EO}: n$ (15-24) with $22 \mathrm{u}$ intervals. The most intense peak at $m / z 577$ is corresponding to the potassiated $\mathrm{C}_{12} \mathrm{EO}: 8$. In a similar fashion, Figure $3 \mathrm{~b}$ exhibited the serial peaks corresponding to the $[\mathrm{M}+3 \mathrm{~K}]^{3+}$ of $\mathrm{C}_{12}$ EO:n (27-43) with ca. $15 \mathrm{u}$ intervals in addition to those of $[\mathrm{M}+2 \mathrm{~K}]^{2+}$ with $22 \mathrm{u}$ intervals, but exhibited less intense peaks of $[\mathrm{M}+\mathrm{K}]^{+}$. Similarly, the mass spectra of $\mathrm{C}_{12} \mathrm{EO}: 40$ (average) cationized by $\mathrm{Li}^{+}$, by $\mathrm{Na}^{+}$, by $\mathrm{Rb}^{+}$, and by $\mathrm{Cs}^{+}$clearly exhibited their respective doubly and triply cationized molecules. However, the triply cesiated molecules were not observed under the 

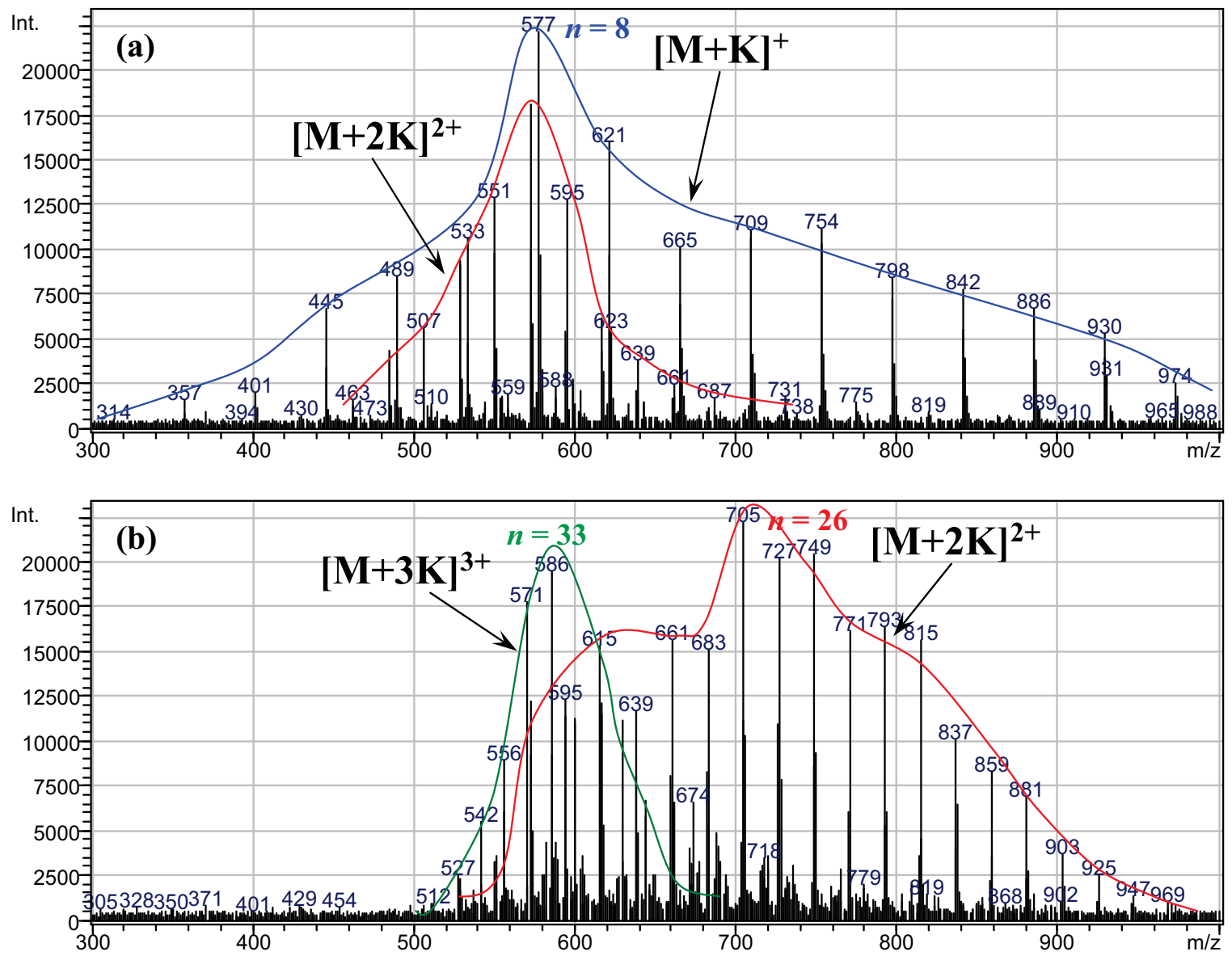

Figure 3. ESI mass spectra of (a) $\mathrm{C}_{12} \mathrm{EO}: 13$ (average) and (b) $\mathrm{C}_{12} \mathrm{EO}: 40$ (average) cationized both by $\mathrm{K}^{+}$. "M" denotes individual $\mathrm{C}_{12} \mathrm{EO}: n$ (average) molecules.

working condition, probably owing to the excessively large ionic size.

We would introduce the critical EO unit numbers necessary to start producing the multiply cationized molecules, which were very dependent on the guest cation diameters as summarized in Table 2. Although the diameter of $\mathrm{Li}^{+}$is significantly smaller than that of $\mathrm{Na}^{+}, \mathrm{Li}^{+}$required almost the same critical $\mathrm{EO}$ unit numbers as $\mathrm{Na}^{+}$needed, i.e., 12 and 24. This is consistent with the estimation that the size selectivity of the helical POE is less specific than that of the cyclic POE. The differential critical unit numbers between the doubly and triply cationized molecules were 12 by $\mathrm{Li}^{+}$and $\mathrm{Na}^{+}, 13$ by K${ }^{+}, 14$ by $\mathrm{Rb}^{+}$, and 15 by $\mathrm{Cs}^{+}$. Since the electrostatic repulsive forces generating between alkali cations seem to be independent of the elements because of the same charge, such additional increase in the EO unit number is probably owing to the increase in ionic size. This can lead to an idea that the POE helix including alkali cations can adjust their helical conformation three-dimensionally to just fit the cation size.

\section{Characteristic Difference in Structure of Protonated POE Surfactants}

Figure 4 shows the mass spectra of (a) $\mathrm{C}_{12} \mathrm{EO}: 13$ (average) and (b) $\mathrm{C}_{12} \mathrm{EO}: 40$ (average) both protonated by adding trifluoroacetic acid as protonation agent. Singly, doubly, and triply charged protonated $\mathrm{C}_{12} \mathrm{EO}: n\left([\mathrm{M}+\mathrm{H}]^{+}\right.$, $[\mathrm{M}+2 \mathrm{H}]^{2+}$, and $[\mathrm{M}+3 \mathrm{H}]^{3+}$ ) were observed through the two mass spectra, but their peak intensities were significantly less than those cationized by alkali-metal ions. This suggests, therefore, that the proton selectivity of the POE surfactants is significantly less than the cation affinity under the ESI conditions. In addition, the

Table 2. Critical EO unit numbers for alkali-metal ions

\begin{tabular}{ccc}
\hline Alkali cation & Double adduct & Triple adduct \\
\hline \hline $\mathrm{Li}^{+}$ & 12 & 24 \\
$\mathrm{Na}^{+}$ & 12 & 24 \\
$\mathrm{~K}^{+}$ & 15 & 28 \\
$\mathrm{Rb}^{+}$ & 17 & 31 \\
$\mathrm{Cs}^{+}$ & 19 & 34 \\
\hline
\end{tabular}



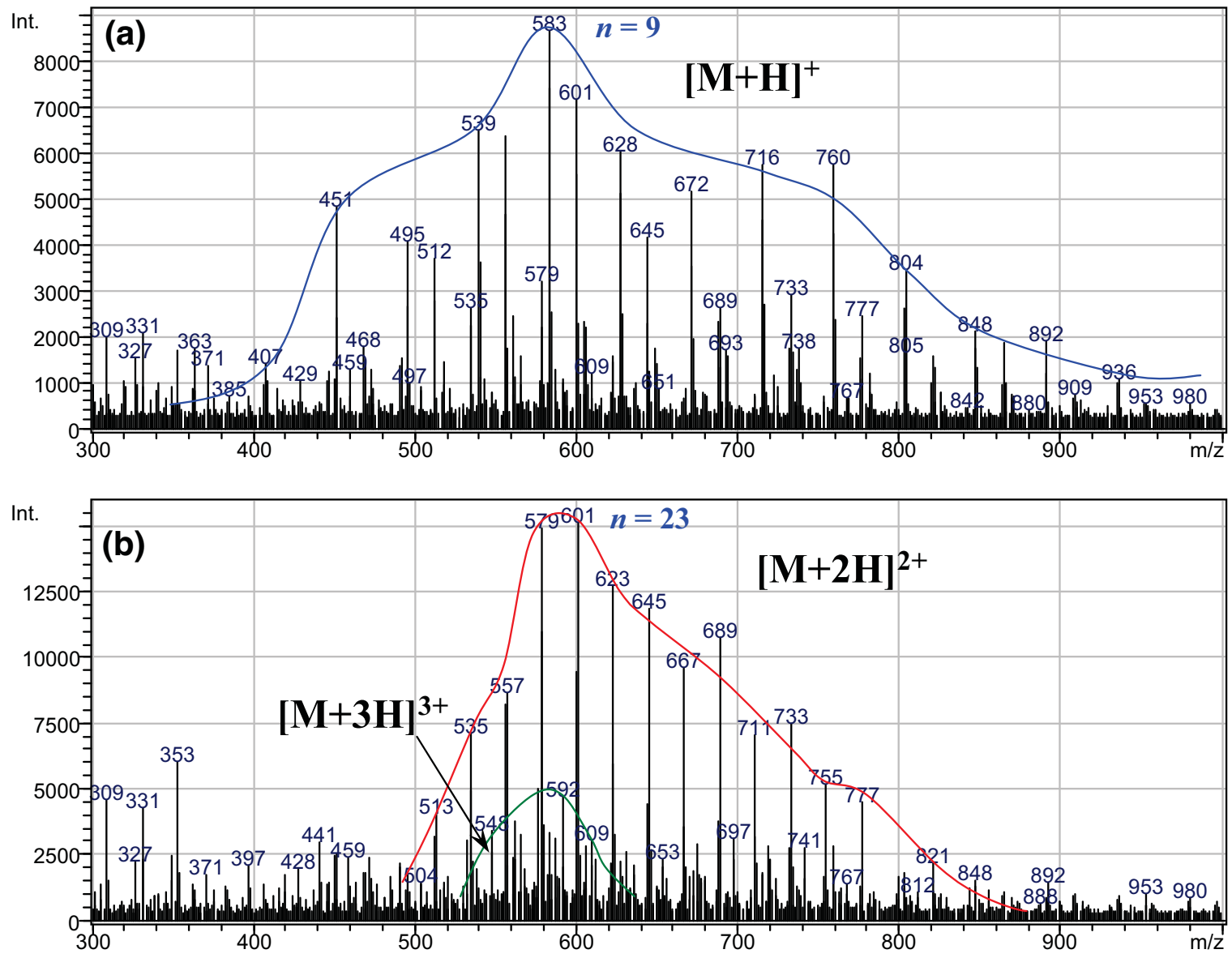

Figure 4. ESI mass spectra of both protonated (a) $C_{12}$ EO:13(average) and (b) $C_{12} E O: 40$ (average).

triply protonated molecules were also observed, but were less abundant probably because of only forming unstable conformations.

The critical EO unit numbers necessary to start producing the doubly and triply protonated molecules were 16 and 30, respectively, although the size of proton is fairly smaller than those of alkali cations. This may suggest that the helical conformation of POE oligomers can be destroyed by additional covalent bonding such as hydrogen bonds to the heteroatoms.

\section{Molecular Mechanics/Dynamics Calculations}

Figure 5 shows an energy optimized gas-phase conformation of $\mathrm{C}_{12} \mathrm{EO}: 15$ including two potassium ions (a) and a snapshot from their molecular dynamics simulation (b). According to the energy optimization, two potassium ions each at an interval were centrically coordinated to six oxygen atoms helically arranged in the POE moiety (Figure 5a). The dodecyl end-group could only place toward outside of the helix and little affect the helical conformation.

On the one hand, the molecular dynamics simulation clearly indicated that $\mathrm{C}_{12} \mathrm{EO}: 15$ could continue to hold two potassium ions in the POE moiety for $200 \mathrm{ps,}$ whereas $\mathrm{C}_{12}$ EO:12 could hold only one potassium ion but could hold two sodium ions. This is very consistent with the critical EO unit numbers described above. The dynamic structures of $\mathrm{C}_{12} \mathrm{EO}: 15$ containing two potassium ions were somewhat different from those obtained through the energy optimization. Since the distance between the two cations was a little extended, the POE moiety with two cations came to take a dumbbell-like conformation. In addition, the POE conformation was independent of the alkyl end-group. The dodecyl endgroup could only approach the intermediate EO moiety of a little stretched helical POE, as shown in Figure 5b, which was probably due to hydrophobic interaction.

Therefore, such theoretical molecular modeling simulations are very indicative of the experimental findings mentioned above. Since one helical turn consisting of 6 EO residues can be recognized in the simulation, the apparent inner diameters of the horizontal section of the helical structure when including $\mathrm{K}^{+}$are thought to be similar to the hole diameter of 18-crown-6.

\section{Conclusions}

Although many articles describing cation affinity of PEGs have been published for the last few decades [13-22], we have first presented the differences in cation selectivity between cyclic and helical POEs, by using 

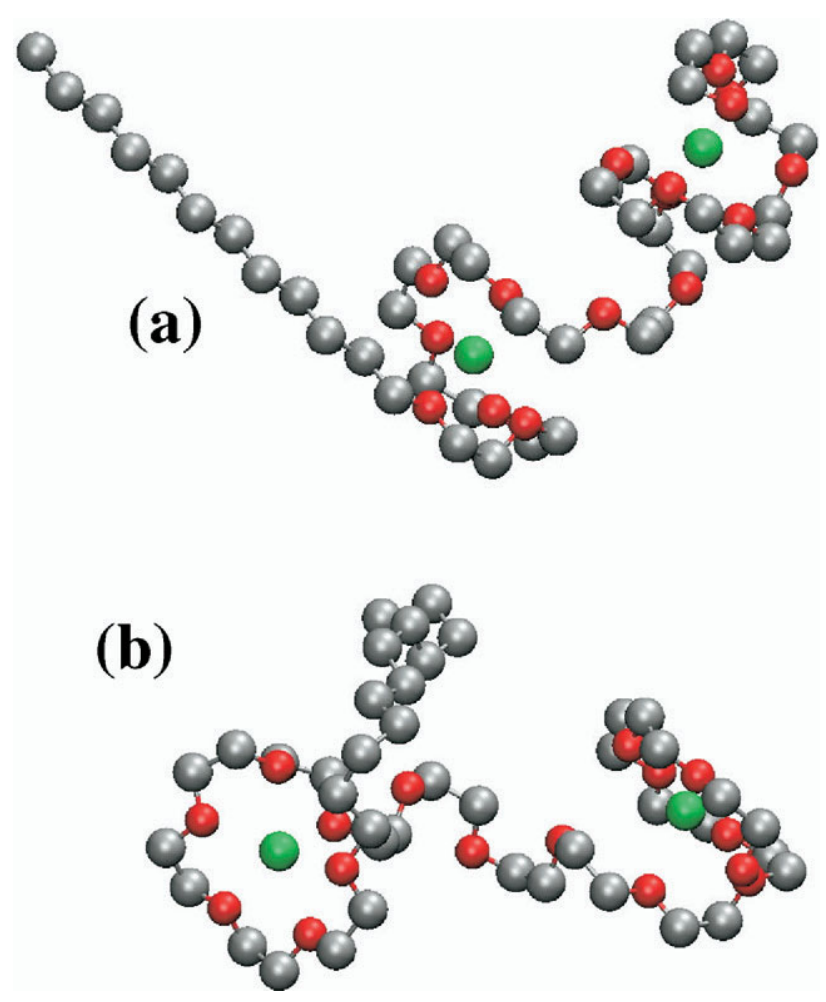

Figure 5. A potential minimum structure (a) and a snapshot (b) from the molecular dynamics simulation for doubly potassiated $\mathrm{C}_{12} \mathrm{EO}: 15$, both depicted orthographically. Carbon atoms are shown in gray, oxygen atoms in red, and $\mathrm{K}^{+}$ion in green.

POE dodecylether nonionic surfactants. The possible host-guest interaction between the helical POE moiety and alkali cations was inferred experimentally and theoretically. The potassiated $\mathrm{C}_{12} \mathrm{EO}: n$ is the most stable in gas-phase, and one helical turn of the POE chain must be comprised of six consecutive oxyethylene units. The critical EO unit numbers introduced by this study can well explain the similar results from electrohydrodynamic ionization of PEGs by Cook et al. [13].

The molecular mechanical/dynamics simulation could provide very helpful results indicative of the POE helical conformations with alkali cation(s). However, the calculated thermochemical data for the protonated molecules were inconsistent with the experimental results; that is, the protonated POE surfactant should be thermodynamically more stable than the alkali-cationized molecules. Such inconsistency may come from the fact that the molecular mechanics/dynamics calculation can simulate chemical interactions without charge migration but cannot demonstrate chemical reactions such as protonation forming a new covalent bond. Therefore, it seems difficult to rationalize thermochemically our experimental findings that the alkali-cationized POE surfactants are more stable than their protonated molecules, which is consistent with the earlier Cook's results [13]. Contrary to this, the molecular modeling simulation has proved that the stability in helical conformations of cationized POE surfactants can arise from the highly size-dependent host-guest interac- tions based on Coulomb and van der Waals forces. From the ionic structure viewpoint, such cationized molecules may be expressed as $\left[\mathrm{M}+\mathrm{K}^{+}\right]$.

\section{References}

1. Yamashita, M.; Fenn, J. B. Electrospray Ion Source. Another Variation on the Free-Jet Theme. J. Phys. Chem. 1984, 88, 4451-4459.

2. Yokoyama, Y.; Fukazawa, Y.; Ito, T.; Sato, H. Identification of Unknown Surfactants Using Electrospray Mass Spectrometry and NMR Spectroscopy Preceded by Liquid Ionization Mass Spectrometry. Spectrochim. Acta A 2002, 58, 1453-1460.

3. Tasaki, K. Poly(Oxyethylene)-Water Interactions: A Molecular Dynamics Study. J. Am. Chem. Soc. 1996, 118, 8459-8469.

4. Pedersen, C. J. Cyclic Polyethers and Their Complexes with Metal Salts. J. Am. Chem. Soc. 1967, 89, 7017-7036.

5. Pedersen, C. J. Crystalline Salt Complexes of Macrocyclic Polyethers. J. Am. Chem. Soc. 1970, 92, 386-391.

6. Pedersen, C. J. New Macrocyclic Polyethers. J. Am. Chem. Soc. 1970, 92, 391-394.

7. Izatt, R. M.; Eatough D. J.; Christensen, J. J. Thermodynamics of Cation-Macrocyclic Compound Interaction. Struct Bonding 1973, 16, 161-189.

8. Izatt, R. M.; Terry R. E.; Haymore, B. L.; Hansen, L. D.; Dalley, N. K.; Avondet, A. G.; Christensen, J. J. Calorimetric Titration Study of the Interaction of Several Uni- and Bivalent Cations with 15-Crown-5, 18-Crown-6, and Two Isomers of Dicyclohexo-18-Crown-6 in Aqueous Solution at $25^{\circ} \mathrm{C}$ and $\mu=0.1$. J. Am. Chem. Soc. 1976, 98, 7620-7626.

9. Hay, B. P.; Rustad, J. R.; Hostetler, C. J. Quantitative Structure-Stability Relationship for Potassium Ion Complexation by Crown Ethers. A Molecular Mechanics and ab Initio Study. J. Am. Chem. Soc. 1993, 115, $11158-11164$

10. Kempen, E. C.; Brodbelt, J. S.; Bartsch, R. A.; Jang, Y.; Kim, J. S. Investigation of Alkali Metal Cation Selectivities of Lariat Ethers by Electrospray Ionization Mass Spectrometry. Anal. Chem. 1999, 71, 54935500 .

11. Blair, S. M.; Brodbelt, J. S.; Marchand, A. P.; Kumar, K. A.; Chong, H.-S. Evaluation of Binding Selectivities of Caged Crown Ligands toward Heavy Metals by Electrospray Ionization/Quadrupole Ion Trap Mass Spectrometry. Anal. Chem. 2000, 72, 2433-2445.

12. Kempen, E. C.; Brodbelt, J. S. A Method for the Determination of Binding Constants by Electrospray Ionization Mass Spectrometry. Anal. Chem. 2000, 72, 5411-5416.

13. Lai, S.-T. F.; Chan, K. W.; Cook, K. D. Electrohydrodynamic Ionization Mass Spectrometry of Poly(Ethylene glycols). Macromolecules 1980, 13, 953-956.

14. Chan, K. W. S.; Cook, K. D. Extended Mass Range by Multiple Charge: Sampling Quadruply Charged Quasimolecular Ions of Poly(Ethylene Glycol) 4000. Org. Mass Spectrom. 1983, 18, 423-425.

15. Wong, S. F.; Meng, C. K.; Fenn, J. B. Multiple Charging in Electrospray Ionization of Poly(Ethylene glycols). J. Phys. Chem. 1988, 92, 546-550.

16. Fenn, J. B.; Mann, M.; Meng, C. K.; Wong, S. F.; Whitehouse, C. M. Electrospray Ionization for Mass Spectrometry of Large Biomolecules. Science 1989, 246, 64-71.

17. Zhou, S.; Cook, K. D. A Mechanistic Study of Electrospray Mass Spectrometry: Charge Gradients Within Electrospray Droplets and Their Influence on Ion Response. J. Am. Soc. Mass Spectrom. 2001, 12, 206-214.

18. Bogan, M. J.; Agnes, G. R. Poly(Ethylene glycol) Doubly and Singly Cationized by Different Alkali Metal Ions: Relative Cation Affinities and Cation-Dependent Resolution in a Quadrupole Ion Trap Mass Spectrometer. J. Am. Soc. Mass Spectrom. 2002, 13, 177-186.

19. von Helden, G.; Wyttenbach, T.; Bowers, M. T. Conformation of Macromolecules in the Gas Phase: Use of Matrix-Assisted Laser Desorption Methods in Ion Chromatography. Science 1995, 267, 1483-1485.

20. Gidden, J.; G.; Wyttenbach, T.; Jackson, A. T.; Scrivens, J. H.; Bowers, M. T. Gas-Phase Conformations of Synthetic Polymers: Poly(Ethylene Glycol), Poly(Propylene Glycol), and Poly(Tetramethylene Glycol). J. Am. Chem. Soc. 2000, 122, 4692-4699.

21. Gidden, J.; Bowers, M. T.; Jackson, A. T.; Scrivens, J. H. Gas-Phase Conformations of Cationized Poly(Styrene) Oligomers. J. Am. Soc. Mass Spectrom. 2002, 13, 499-505.

22. Jackson, A. T.; Scrivens, J. H.; Williams, J. P.; Baker, E. S.; Gidden, J.; Bowers, M. T. Microstructural and Conformational Studies of Polyether Copolymers. Int. J. Mass Spectrom. 2004, 238, 287-297.

23. Brooks, B. R.; Bruccoleri, R. B.; Olafson, B. D.; States, D. J.; Swaminathan, S.; Karplus, M. CHARMM: A program for Macromolecular Energy, Minimization, and Dynamics Calculations. J. Comput. Chem. 1983, 4 187-217.

24. Pauling, L. The Sizes of Ions and the Structure of Ionic Crystals. J. Am. Chem. Soc. 1927, 49, 765-790.

25. Vögtle, F. Supramolecular Chemistry: An Introduction; John Wiley and Sons: Chichester, England, 1989; Japanese Edition, Maruzen, Japan, 1995, pp 38.

26. Greene, R. N. 18-Crown-6: A Strong Complexing Agent for Alkali Metal Cations. Tetrahedron Lett. 1972, 18, 1793-1796. 